\title{
Monitoring in real time the effect of TLX overexpression on proliferation and migration of $\mathrm{C} 6$ cells
}

\author{
G. L. $\mathrm{LI}^{1}$, S. H. FANG ${ }^{2}$, B. XU $\mathrm{U}^{1, *}$
}

${ }^{1}$ Department of Neurology, the Second Affiliated Hospital of Zhejiang Chinese Medical University, 318 Chaowang Road, Hangzhou 310005, Zhejiang Province, China; ${ }^{2}$ Core Facilities of Zhejiang University Institute of Neuroscience, 866 Yuhangtang Road, Hangzhou 310058, Zhejiang Province, China

*Correspondence: xubin2008happy@163.com

Received May 22, 2016 / Accepted August 12, 2016

\begin{abstract}
Orphan nuclear receptor TLX has been shown to play an essential role in regulating the self-renewal and proliferation of neural stem cells (NSCs). However, TLX overexpression in NSCs induces long-term NSC expansion and further leads to glioma initiation in mouse when combined with p53 mutations. Whether overexpression of TLX plays a role in glioma stem cell (GSC) proliferation and migration still remains largely unknown. In this study, we infected C6 cells, a special glioma cell line which is mainly composed of cancer stem cells(CSCs), with lentiviruses expressing GFP(LV-GFP) or GFP-T2ATLX(LV-TLX) and then monitored cell proliferation and migration using the real-time analyzer system (RTCA, xCELLigence, Roche). We found that the cell index (CI) observed for the TLX overexpressing C6 cells showed a lower value than that of the LV-GFP transduced cells. And the MTT results correlated highly with the RTCA proliferation assessments. Furthermore, the expression of p21 was decreased while other downstream genes PTEN and p53 were not significantly changed in TLX overexpressing C6 cells. These findings strongly indicate that TLX overexpression has the ability to decrease the proliferating and migratory properties of C6 cells by targeting p21. Further, our results suggest that TLX overexpression may also have a similar inhibitory effect on GSC proliferation and migration.
\end{abstract}

Key words: TLX overexpression, C6 cells, proliferation, migration, $p 21$

TLX (also known as NR2E1) is an orphan nuclear receptor expressed in vertebrate forebrains and highly expressed in the adult brain [1]. It has been identified as a crucial regulator that is involved in both maintaining NSC self-renewal and regulating adult neurogenesis $[2,3,4,5]$. TLX represses its downstream target genes by complexing with histone deacetylases and the lysine-specific histone demethylase LSD1,thus maintaining the undifferentiated characteristics and proliferative potential of NSCs [6]. TLX-null NSCs isolated from adult TLX-mutant mice fail to self-renew and proliferate [4]. On the contrary, NSC-specific overexpression of TLX induces NSC long-term expansion and initiates glioma-like lesions which progress to invasive gliomas when p53 function is additionally inactivated [7].

GSCs are characterized by the ability to generate a clone and subclones which could give rise to a xenograft glioma in vivo [8]. Gliomas have long been suggested originate from the SVZ [9], where new neurons are generated from NSCs. GSCs and
NSCs share many similarities, including the capacity to form spheres under certain culture conditions, the characteristics of extensive self-renew and multipotency, and the expression of nestin and CD133 [10]. These similarities suggest that important regulators of NSCs may play a similar role in GSCs. Consist with this, downregulation of TLX reduces GSC selfrenewal and tumorigenesis [11].

The xCELLigence RTCA system is an alternative noninvasive and label-free advanced technology, which allows continuous real-time monitoring of cell proliferation, migration and invasion on a cell culture level [12]. The xCELLigence system uses custom-designed plates, which are equipped with gold microelectrodes in the bottom of each well. Cells adhere to or detach from the surface electrodes will influence the electrical impedance across the array, which is measured and displayed as cell index (CI) values by the xCELLigence software. The CI value is a dimensionless parameter which can represent variations in cell number, cellular viability and 
morphology as well as adhesion degree. Briefly, when cells are not adhered, CI is zero, and as cells adhere to the array, the CI increases. However, the addition of a cytotoxic inducing agent would decrease the CI value $[13,14]$.

Our original intention was to explore the effect of TX overexpression on GSC proliferation and migration using RTCA system in this study. But we did not have ability to isolate and purify GSCs due to limitations of our experimental methods. As Zheng et al. have demonstrated that most C6 cells are cancer stem cells using clonal and population analyses [8], so we first performed the experiment on $\mathrm{C} 6$ glioma line to assess the role for TLX overexpression in C6 cell proliferation and migration.

\section{Materials and methods}

Reagents. Thiazolyl blue tetrazolium bromide, DMSO (Dimethyl sulfoxide) and polybrene were purchased from Sigma-Aldrich (St Louis, MO, USA). DMEM (Dulbecco's Modified Eagle's medium) and fetal calf serum were purchased from Gibco (Paisley, United Kingdom). The primers used for RT-qPCR were designed and synthesized by Sangon Biotech Co., Ltd (Shanghai, China). All of the primary and second antibodies used in the Western blot were purchased from Beyotime Institute of Biotechnology (Shanghai, China).

Cell culture. Rat C6 glioma cell line was purchased from the Institute of Cell Biology, Chinese Academy of Sciences (Shanghai, China). Cells were maintained in DMEM supplemented with $10 \%$ bovine calf serum and $100 \mathrm{U} / \mathrm{ml}$ penicillin/ streptomycin, at $37^{\circ} \mathrm{C}$ in a humidified atmosphere containing $5 \% \mathrm{CO}_{2}$.

Lentiviral production and transduction. Two lentiviral vectors were used: a green fluorescent protein (GFP)-encoding control vector named GFAP-GFP and a GFP-and TLX-encoding vector named GFAP-GFP-T2A-TLX. Methods used for lentivirus production have been previously described $[3,15]$. The lentiviral vectors and packaging plasmids were transfected into HEK293T cells. Virus-containing supernatants were harvested at 24,48 and $72 \mathrm{~h}$ post transfection, and were subsequently sterile filtered. Lentiviral particles were then concentrated by ultracentrifugation at $112000 \mathrm{~g}$ for $2 \mathrm{~h}$ at $4{ }^{\circ} \mathrm{C}$.

C6 cells were seeded into 6-well plates at a density of $2 \times 10^{5}$ cells/well 1 day prior to lentivirus infection. After overnight culture, the cells were transfected with $4 \mu \mathrm{l}$ virus (each with an original titer of $0.5-2 \times 10^{9}$ colony-forming units per milliliter) in the presence of $8 \mu \mathrm{g} / \mathrm{ml}$ polybrene. Twenty-four hours after transduction, the media were replaced with fresh $10 \%$ FBS containing medium, and the infected cells were incubated for another $48 \mathrm{~h}$. The transfection efficiency was evaluated by observation of green fluorescence protein (GFP) positive cells using a fluorescence microscope. The LV-GFP group and LV-TLX group were transfected with LV-GFP and LV-TLX, respectively, and the blank control group was given the same volume of culture solution.
RTCA assessment. Cell proliferation experiments were performed on the xCELLigence DP device, which was placed in a humidified incubator at $37^{\circ} \mathrm{C}$ and $5 \% \mathrm{CO}_{2}$. Initially, $50 \mu \mathrm{L}$ of cell-free growth medium (10\% FBS) was added in each well of the E-16 plates and then the plates were incubated at room temperature for $30 \mathrm{~min}$. After this incubation period, the base-line impedance was measured to ensure that all the connections were in good condition. The transduced cells were harvested, counted, and were then re-suspended in culture medium. A volume of $100 \mu \mathrm{L}$ cell suspension $\left(2 \times 10^{5} \mathrm{cell} / \mathrm{mL}\right)$ was added to each well of the E-16 plates and the cells were maintained at room temperature for $30 \mathrm{~min}$ to allow cell attachment. Finally, the plates were placed in the xCELLigence system to record the Cell index (CI) every $15 \mathrm{~min}$, for $72 \mathrm{~h}$.

Cell migration experiments were performed using CIM16 plates with each well consisting of an upper and a lower chamber. Firstly, $165 \mu \mathrm{L}$ cell culture medium (10\%FBS) and $30 \mu \mathrm{L}$ serum free medium were added to the lower and upper chambers, respectively and the plates were placed on the RTCA DP Station for $1 \mathrm{~h}$ to obtain equilibrium. Then the plates were connected to the system to measure background impedance. Following harvesting and counting, 40,000 infected cells/well suspended in $100 \mu \mathrm{L}$ serum free medium were seeded in the upper chamber. After leaving the plates at room temperature for $30 \mathrm{~min}$, they were monitored in an xCELLigence DP platform for $24 \mathrm{~h}$ with impedance recorded at intervals of 15 min.

MTT assay. The MTT assay is a colorimetric technique used to analyze cell proliferation and mitochondrial function. After lentiviral transduction, cells in each group were digested and counted, then cultured in 96 -well plates at $2 \times 10^{4}$ cells/well for specific time periods $(24,48$ and $72 \mathrm{~h})$. Subsequently, $20 \mu \mathrm{L}$ MTT $(5 \mathrm{mg} / \mathrm{mL})$ solution was added to each well , and the cells were cultured for 2 hours at $37^{\circ} \mathrm{C}$. The supernatants were carefully removed and $100 \mu \mathrm{l}$ of DMSO was added per well. And then the plates were shaken for 10 min until the purple crystals were completely dissolved. The absorbance value at $570 \mathrm{~nm}$ was read using a microplate reader (Elx800, Bio-TEK Instrument USA).

RNA isolation and qPCR. Total RNA from transduced C6 cells was extracted using TRIzol reagent (Invitrogen) according to the manufacturer's protocol. Total RNA purity and concentration were determined by measuring A260:A280 ratios using a NanoDrop 2000 spectrophotometer (Thermo Scientific). A ratio close to 2.0 was considered high purity. And reverse transcription was performed to obtain cDNA using a PrimeScript reverse transcriptase reagent kit (TaKaRa) in accordance with the manufacturer's instructions. The expression levels of TLX gene, PTEN gene, P53 gene, and P21 gene were determined using a LightCycler 480 (Roche). A house-keeping gene GAPDH (glyceraldehyde-3-phosphate dehydrogenase) was used as an internal control to normalize samples. Gene-specific primers used were as follows: TLX,(forward) 5'-TGGACACAAGGAAGACAATGG-3' and (reverse) 5'-GACAAAGTGGAAAAGGCTGG-3- 
';PTEN,(forward)5' - GGAAAGGACGGACTGGTGTA-3' and (reverse) 5'-TGCCACTGG TCTGTA ATCCA-3';P53, (forward) 5'-TCTGTCATCTTCCGTCCCTTCTC-3' and (reverse) 5'-CCGTGCACATAACAGACTTGGCT-3';P21, (forward) 5' - GTTCCT TGCCACTTCTTAC -3'and (reverse) 5' - ACTGCTTCACTGTCATCC -3';GADPH, (forward) 5'-GGCAAGTTCAACGGCACAG-3' and (reverse) 5'CGCCAGTAGACTCCACGACAT-3'. RT-qPCR reactions were performed in a $10 \mu \mathrm{l}$ final volume containing $5 \mu \mathrm{l}$ SYBR Premix Ex Taq (TaKaRa), $0.2 \mu$ l of each forward and reverse gene-specific primer $(5 \mu \mathrm{M}), 0.6 \mu$ sterile distilled water and $4.0 \mu \mathrm{l}$ of cDNA(1:40dilution). The relative amount of messenger RNA (mRNA) in each target gene to GAPDH was calculated using the ${ }^{\Delta \Delta} \mathrm{Ct}$ method. ${ }^{\Delta \Delta} \mathrm{Ct}$ method: ${ }^{\Delta} \mathrm{Ct}=\mathrm{Ct}$ target $-\mathrm{Ct}_{\mathrm{GAPDH}},{ }^{\Delta} \mathrm{Ct}={ }^{\Delta} \mathrm{Ct} \mathrm{treated}-{ }^{\Delta} \mathrm{Ct}$ control and gene expression is reported as $2^{-\Delta \Delta \mathrm{Ct} \text {. }}$

Western blot. After lentiviral transfection, cells were lysed and the protein concentrations were determined by the Bradford method using bovine serum albumin (BSA) as a standard. Sample proteins (100 $\mu \mathrm{g}$ per lane) were electrophoresed on $12 \%$ SDS-PAGE and transferred to nitrocellulose membranes. Membranes were blocked in 5\% fat-free milk for $1 \mathrm{~h}$ at room temperature and then incubated with primary antibodies against PTEN, p53, p21 and GAPDH overnight at $4{ }^{\circ} \mathrm{C}$. GAPDH was used as a loading control. After incubation with appropriate secondary antibodies for $2 \mathrm{~h}$ at room temperature, Odyssey infrared imaging system (LI-COR Biosciences) was used to visualize the protein bands. The densities of the Western blot bands were measured using ImageJ software.

Statistical analysis. Statistical analysis was performed using GraphPad Prism 6.0 (GraphPad Software, Inc., USA). All quantitative data are expressed as means \pm standard deviations, and analyzed by one-way analysis of variance (ANOVA). $\mathrm{P}<0.05$ was considered to indicate a statistically significant difference.

\section{Results}

Lentiviral vectors effectively infected $c 6$ cells and overexpressed TLX. After transduction of C6 cells by the LV-GFP and the LV-TLX, the transduction efficiency were determined at day 3 post transduction by counting the percentage of GFP positive cells within a transduced cell population using a fluorescence microscope. As shown in Figure 1A, more than 80\%
A
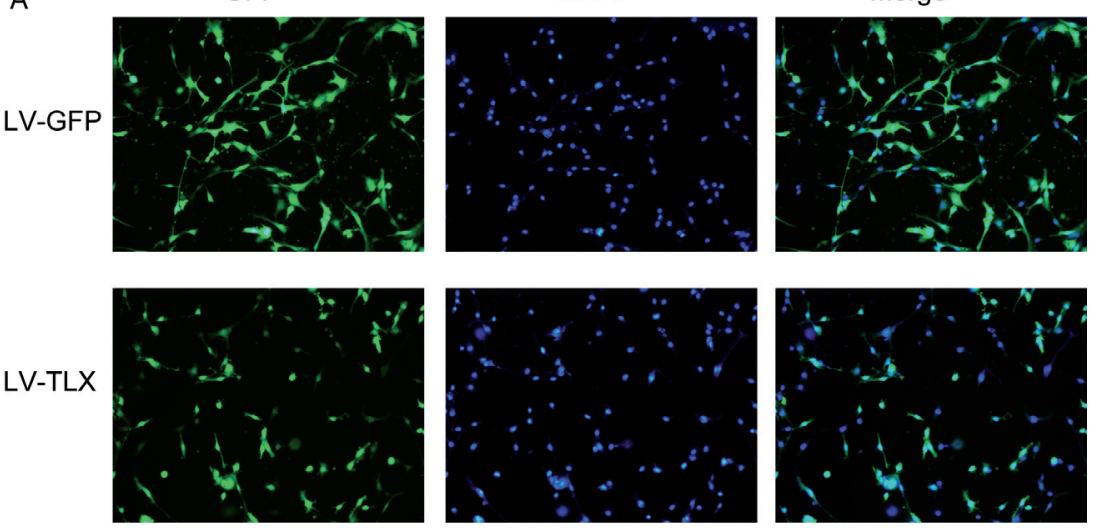

B

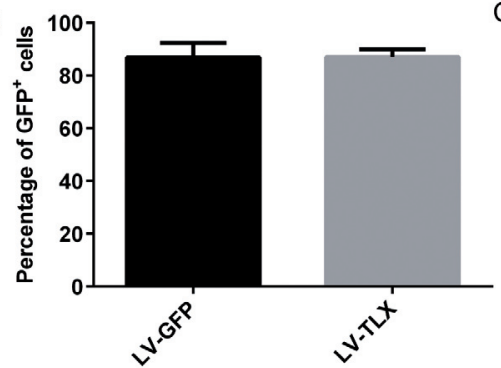

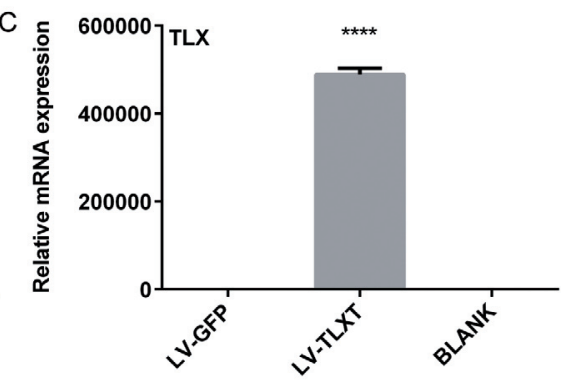

Figure 1. Lentiviral vectors effectively infected C6 cells and overexpressed TLX. (A) Representative microscopic images of lentiviral vector transduced C6 cells showing GFP expression. 72h after lentiviral transduction, GFP expressions in the infected cells were evaluated using a fluorescence microscope (original magnification $\times 20$ ). Nuclei were stained with DAPI for identification of the cells. (B) Transduction efficiency of transduced cells for LV-GFP and LV-TLX (bar: mean \pm SEM; $\mathbf{n}=6$ ). The transduction efficiencies between different vectors were not significant. (C) Relative TLX expression in C6 cells was expressed as the mean fold change \pm standard deviation compared with the LV-GFP group. 
cells transfected with the LV-GFP and LV-TLX were positive for GFP, indicating a high transduction efficiency of both lentiviral vectors. And no significant difference in transduction efficiency was observed between the LV-GFP and LV-TLX vectors, displaying transduction efficiency of $86.9 \pm 5.4 \%(\mathrm{n}=$ $6)$, and $87.1 \pm 2.8 \%(\mathrm{n}=6)$, respectively (Figure $1 \mathrm{~B})$.

To further validate the microscopy data, the expression of TLX transgene in transduced cell was quantified by qPCR after transduction. As shown in Figure 1C, the expression of TLX was found to be significantly higher in the LV - TLX transduced cells than the LV-GFP infected cells. However, no significant change of TLX expression was found between the LV-GFP group and blank control group.

Real time analysis of proliferation and migration of $\mathrm{C} 6$ cells in the LV-GFP group, LV-TLX group and blank control group. To investigate the role of TLX overexpression in the proliferation of $\mathrm{C} 6$ cells and determine the changing over time, we employed RTCA experiments on LV-GFP, LV-TLX transduced and uninfected C6 cells (five wells for each group). As assessed by the xCELLigence platform, the LV-TLX transfected cells displayed a decreased proliferation rate compared to the LV-GFP infected cells, and the reduction in CI became more significant over time (Figure 2A). Figure $2 \mathrm{~B}$ is a representation of interval calculated doubling time that is determined by the RTCA software. Doubling time of the LV-TLX group showed significant increase compared to that of the LV-GFP group between the 48 and $72 \mathrm{~h}$ interval, indicating that cell proliferation of the LV-TLX group decreased significantly. Together, these results show a significant inhibitory effect of TLX overexpression on proliferation of C6 cells.

Regarding migration assay, the RTCA results showed that the CI values of the LV-TLX group reached a lower level than that of the LV-GFP group, suggesting that migratory capacity of TLX overexpressing cells was impaired (Figure 2C). We also calculated the slope of curves between the 0 and $8 \mathrm{~h}$ interval for Figure $2 \mathrm{C}$ and found significant difference between the LV-TLX and LV-GFP group. Lower slope values of the LV-TLX group further indicated that TLX overexpression inhibits the migration of C6 cells. We also observed a low proliferation and migration rate of the
A

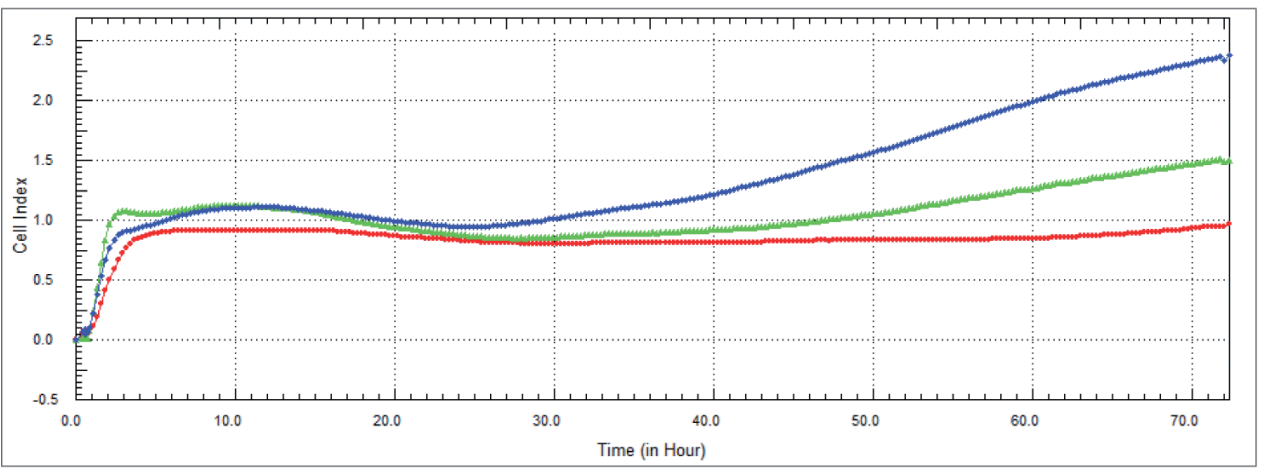

C

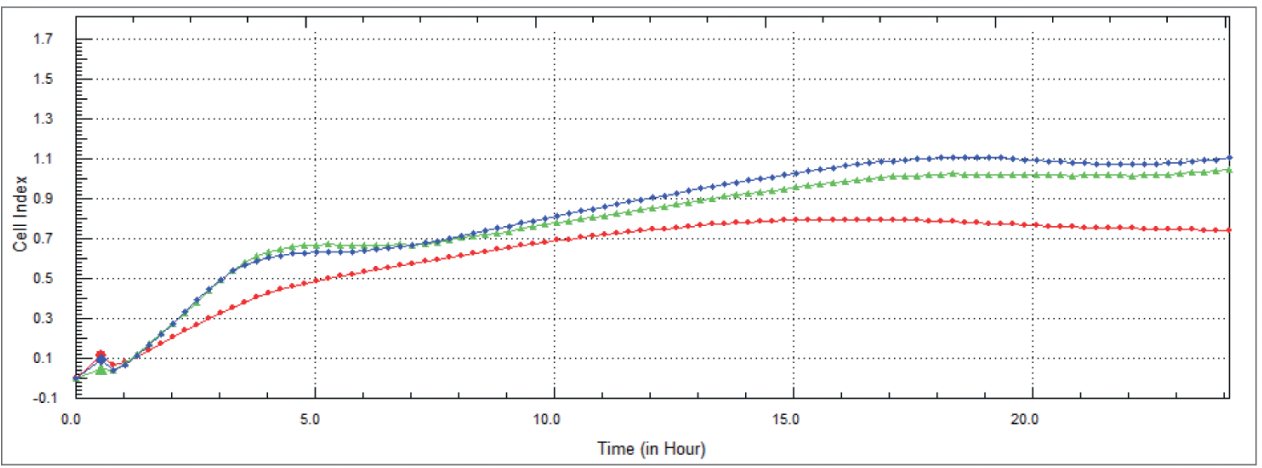

B

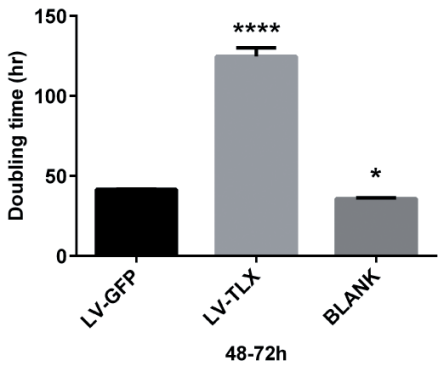

D

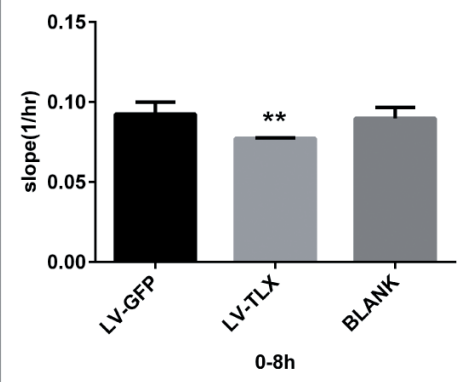

Figure 2. Dynamic assessment of cell proliferation and migration using RTCA. (A) Representative graph comparing the cell proliferation rates of the LV-GFP group (green curve), LV-TLX group (red curve) and blank control group (blue curve). (B) The analysis of the doubling time of curves between the 48 and 72h interval for Figure 2A. (C) Representative graph comparing the cell migration rates of the LV-GFP group (green curve), LV-TLX group (red curve) and blank control group (blue curve). (D)The analysis of the slope of curves between the 0 and $8 \mathrm{~h}$ interval for Figure $2 \mathrm{C}$. The data were represented as mean $\pm S D$. ${ }^{\star} P<0.05,{ }^{* *} \mathrm{P}<0.01$ and ${ }^{\star * *} \mathrm{p}<0.001$, compared with the LV-GFP group $(\mathrm{n}=5)$. 
LV-GFP group compared to that of the blank control group, this may be due to the toxicity of lentivirus and is inevitable (Figure 2A and C).

Overexpression of TLX inhibited the proliferation of C6 cells. The results from MTT assay demonstrated that the LV-TLX infected cells exhibited significant decrease in the cell proliferation compared to the LV-GFP group at three different time points (Figure 3A-C). Though the growth rates of LV-GFP transfected cells were significantly decreased as compared with that of the blank control group at 48 and $72 \mathrm{~h}$, this may be caused by toxicity of lentivirus (Figure 3B and C). We also generated cell proliferation curves based on the optical density (OD) values, which indicated that the inhibition of TLX overexpression on cell growth become more evident over time (Figure 3D). All these suggested that the cell proliferation is significantly suppressed after transfection with LV-TLX. Interesting, the growth curve based on OD measurements showed a similar pattern when compared with the proliferation curve generated from xCELLigence data, implying that both methods detect similar process kinetics (Figure 3D and 2A).

Effects of TLX overexpression on PTEN, p53 and p21 expression in $\mathrm{C6}$ cells. The tumor suppressor genes PTEN, p53 and the Cip/Kip family cyclin-dependent kinase inhibitor p21 have been identified as TLX downstream targets [3,
$4,15]$. We further asked whether these targets participate in the inhibitory effect of TLX overexpression on cell proliferation and migration. Data from qPCR analysis showed that the relative mRNA level of p21 in LV-TLX group was decreased significantly compared to LV-GFP group (Figure 4C). However, PTEN and p53 expression did not exhibit significant change between these two groups (Figure $4 \mathrm{~A}$ and $\mathrm{B}$ ).

Next, we used the Western blot assay to further evaluate whether TLX overexpression caused suppression of p21 expression. As depicted in Figure 5, the p21 protein level in the LV-TLX group exhibited a significant decrease as compared with the LV-GFP group (Figure 5D). While TLX overexpression did not significantly change the PTEN (Figure 5B) and p53 (Figure 5C) protein levels, which is in agreement with the result from qPCR. In addition, we could not detect any significant change in the mRNA and protein expressions of all three genes between the LV-GFP group and blank control group (Figure 4 and Figure 5). Taken together, these results indicate that TLX overexpression inhibited C6 cell proliferation and migration via downregulation of $\mathrm{p} 21$.

\section{Discussion}

Similarities between NSCs and GSCs support the idea that TLX, the critical regulator of NSC self-renewal, may also
A

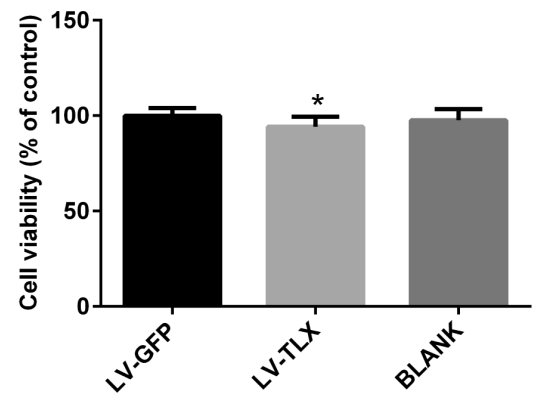

$24 h$

C

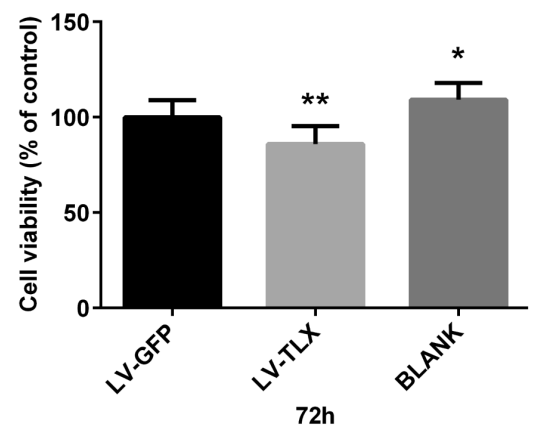

B

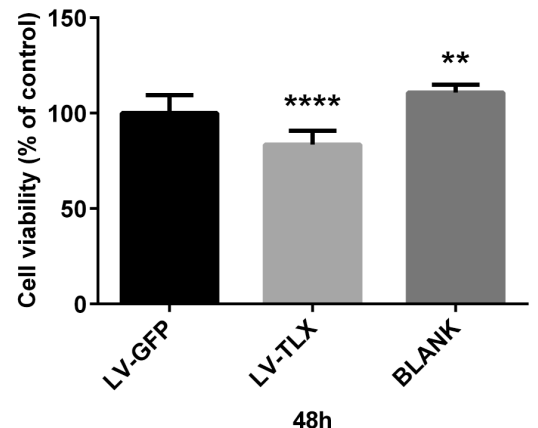

D

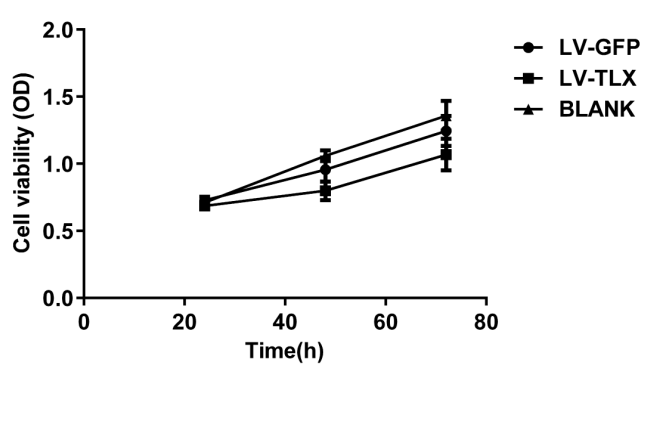

Figure 3. MTT assay revealed that TLX overexpression inhibited C6 cell proliferation. Cell viability of each group at $24 \mathrm{~h}(\mathrm{~A})$, $48 \mathrm{~h}(\mathrm{~B})$, and $72 \mathrm{~h}(\mathrm{C})$ expressed in percentage. The data were represented as mean $\pm \mathrm{SD}$. ${ }^{\star} \mathrm{P}<0.05,{ }^{\star \star} \mathrm{P}<0.01$ and ${ }^{\star * \star} \mathrm{p}<0.001$, compared with the $\mathrm{LV}$-GFP group ( $\left.\mathrm{n}=12\right)$. (D) The cell proliferation curves based on the OD values in each group showed that cell proliferation decreased in the LV-TLX group. 
A

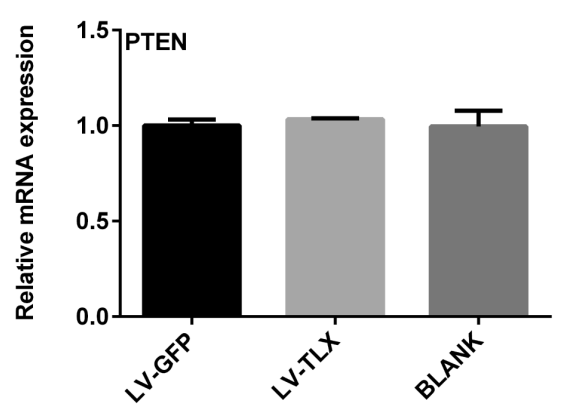

B

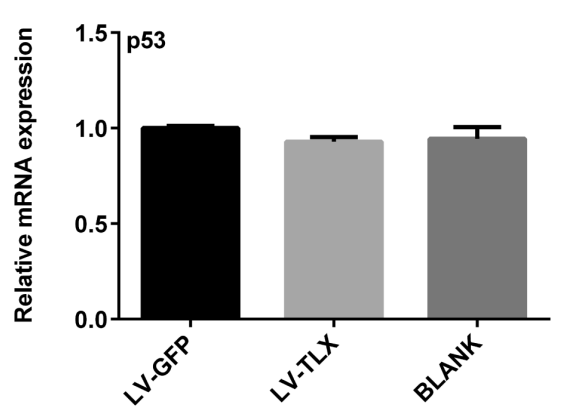

C

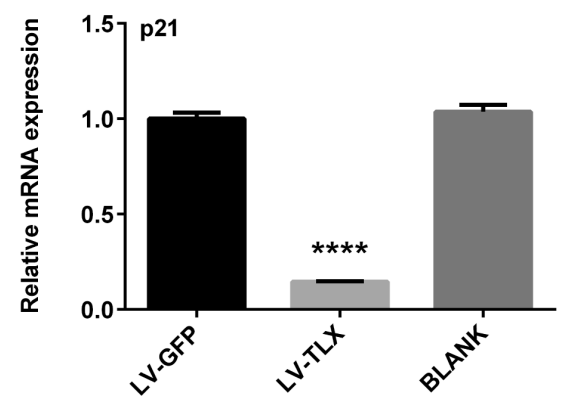

Figure 4. qPCR analysis of TLX target genes. The mRNA levels of PTEN (A), p53 (B), and p21(C) were each normalized against the expression of GAPDH and expressed as the mean fold change \pm standard deviation compared with the LV-GFP group. ${ }^{\star} P<0.05,{ }^{* *} \mathbf{P}<0.01$ and ${ }^{\star * *}$ p $<0.001$, compared with the control.

play an important role in GSCs. However, the role for TLX overexpression in GSCs remain poorly understood while TLX overexpression in NSCs has been proven to be sufficient to cause long-term NSC expansion and initiate the gliomas[7]. The present study demonstrates that overexpression of TLX inhibited cell proliferation and migration via suppressing p 21 in C6 cells, a glioma cell line which is mainly composed of cancer stem cells.

A

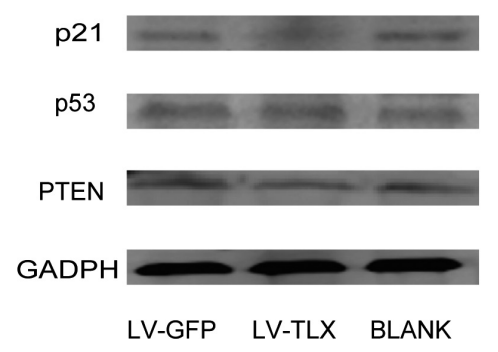

C

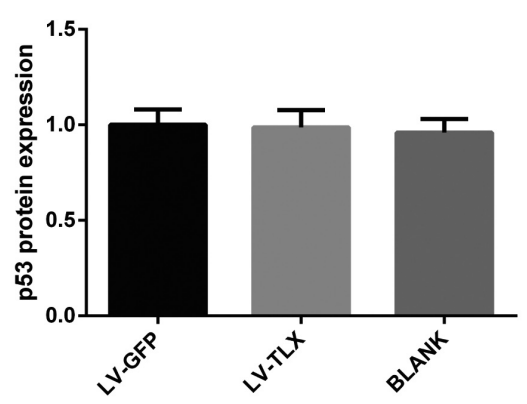

The main properties of stem cells include multilineage developmental potential and long-term self-renewal. It has been described that stem cells have extensive proliferative capacity to perpetuate themselves. However, several genetic models have suggested that increased proliferation results in exhaustion of stem cell function. Consist with this, the proliferation capacity of $\mathrm{p} 21^{-1}$ hematopoietic stem cells (HSCs) is reduced following prior expansion [16] and p21 loss leads to
B

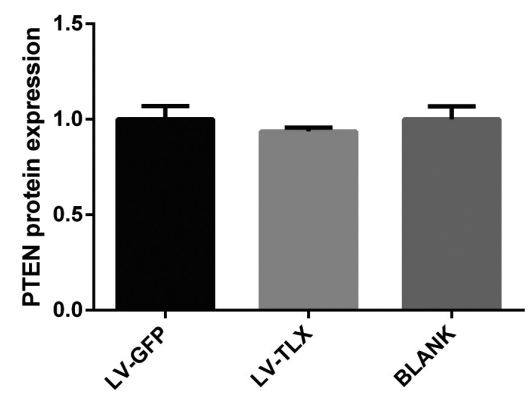

D

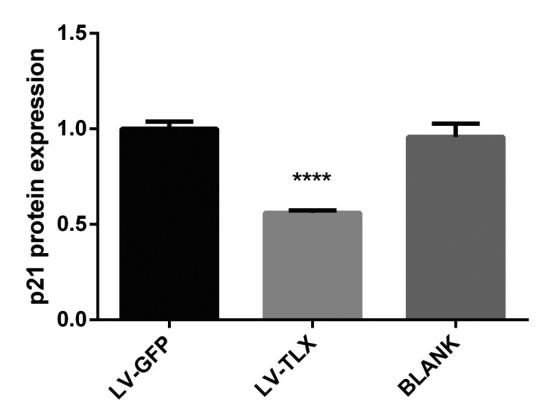

Figure 5. TLX overexpression represses p21 expression at the protein level. (A) Representative Western blot of three independent experiments. (B-D) Quantitative analysis of three independent experiments. The densities of PTEN (B), p53 (C), and p21 (D) bands were measured, normalized to the loading control (GAPDH) and ratios were corrected to the LV-GFP group to quantify relative changes. ${ }^{\star} \mathrm{P}<0.05,{ }^{\star \star} \mathrm{P}<0.01$ and ${ }^{\star * *} \mathrm{p}<0.001$, compared with the LV-GFP group. 
an initial expansion of adult NSCs followed by a diminished NSC maintenance capacity both in vivo and in vitro [17]. HSCs that are null for PTEN show a similar phenotype to that of $\mathrm{p} 21^{-/} \mathrm{HSCs}$ with regard to cell cycling and exhaustion [18]. As with the preceding models, inducible deletion of Foxo1, 3 and 4 also leads to a reduction in HSC function [19]. These studies show that an initial increase in stem cells expansion results in a decrement in long-term maintenance of stem cells, demonstrating that stem cells have a limited repopulation potential. We suggest that $\mathrm{C} 6$ cells may share this property with other stem cells as most C6 cells possess the main defining characteristics of cancer stem cells [8]. Therefore, we speculated that TLX overexpression initially increased C6 cell proliferation and migration based on the essential role for TLX in gliomagenesis [7, 11]. However, increased proliferation leads to exhaustion of cell function which finally results in declined proliferation and migration rates in RTCA assessment (Figure 2A and C). The reason why RTCA assessment failed to reflect any increased proliferation may be that we performed RTCA experiments 3 days after transduction while that increased effect was transient.

The Cip/Kip family cyclin-dependent kinase inhibitor p21 is crucial for maintaining stem cell quiescence and self-renewal. The absence of p21 impairs self-renewal of primitive cells in serially transplanted bone marrow, leading to stem cell exhaustion [16]. Loss of p21 also decreases NSC longevity which is accompanied by progressive decline in proliferation rates [17]. And a recent study reports that $\mathrm{p} 21$ deficiency results in a substantial reduction in HSCs under conditions of cellular stress [20]. p21 is an important target of TLX and is upregulated in TLX null NSCs $[3,15]$. TLX recruits histone deacetylases (HDACs) to the promoter of $\mathrm{p} 21$ to repress its expression . TLX is shown to interact with both HDAC3 and HDAC5 in NSCs [6]. Another mechanism underlying the repression activity of TLX is through the recruitment of lysine-specific demethylase 1 (LSD1). The interaction of TLX and LSD1 results in repression of p21 expression in NSCs [21]. Consistently, we found p21 expression was significantly decreased in TLX-overexpressing C6 cells (Figure 4C and 5D). Therefore, the p21-deficient $\mathrm{C} 6$ cells displayed progressive reduction in proliferation and migration rates under exposure of lentivirus (Figure 2A and C, Figure 3D).

In conclusion, our results shows that TLX overexpression is diverted from tumor promotion in NSCs to tumor inhibition in C6 cells via downregulating p21, a critical regulator of stem cell exhaustion. Considering that most C6 cells are cancer stem cells, it is reasonable to predict an inhibitory role for TLX in GSC proliferation and migration. Future studies will be focused on determining the role of TLX overexpression in other brain tumor stem cells (BTSCs) including GSCs and uncovering the molecular mechanisms underlying the effects of TLX overexpression in BTSCs.

Acknowledgements: This work was supported by the Natural Science Foundation of Zhejiang Province (LY14H090010), China.

\section{References}

[1] YU RT, MCKEOWN M, EVANS RM, UMESONO K. Relationship between Drosophila gap gene tailless and a vertebrate nuclear receptor Tlx. Nature 1994; 370: 375-379. https:/doi. org/10.1038/370375a 0

[2] LIU HK, BELZ T, BOCK D, TAKACS A, WU H et al. The nuclear receptor tailless is required for neurogenesis in the adult subventricular zone. Genes Dev 2008; 22: 2473-2478. https:/doi.org/10.1101/gad.479308

[3] NIU W, ZOU Y, SHEN C, ZHANG CL. Activation of postnatal neural stem cells requires nuclear receptor TLX. J Neurosci 2001; 31: 13816-13828. https:/doi.org/10.1523/ INEUROSCI.1038-11.2011

[4] SHI Y, CHICHUNG-LIE D, TAUPIN P, NAKASHIMA K, RAY J et al. Expression and function of orphan nuclear receptor TLX in adult neural stem cells. Nature 2004; 427: 78-83. https:/doi.org/10.1038/nature02211

[5] ZHANG CL, ZOU Y, HE W, GAGE FH, EVANS RM. A role for adult TLX-positive neural stem cells in learning and behaviour. Nature 2008; 451: 1004-1007. https:/doi.org/10.1038/ nature 06562

[6] SUN G, YU RT, EVANS RM, SHI Y. Orphan nuclear receptor TLX recruits histone deacetylases to repress transcription and regulate neural stem cell proliferation. Proc Natl Acad Sci U S A 2007; 104: 15282-15287. https:/doi.org/10.1073/ pnas.0704089104

[7] LIU HK, WANG Y, BELZ T, BOCK D, TAKACS A et al.The nuclear receptor tailless induces long-term neural stem cell expansion and brain tumor initiation. Genes Dev 2010; 24: 683-695. https:/doi.org/10.1101/gad.560310

[8] ZHENG X, SHEN G, YANG X, LIU W. Most C6 cells are cancer stem cells: evidence from clonal and population analyses. Cancer Res 2007; 67: 3691-3697. https:/doi.org/10.1158/00085472.CAN-06-3912

[9] SANAI N, ALVAREZ-BUYLLA A, BERGER MS. Neural stem cells and the origin of gliomas. N Engl J Med 2005; 353: 811-822. https:/doi.org/10.1056/NEJMra043666

[10] VESCOVI AL, GALLI R, REYNOLDS BA. Brain tumour stem cells. Nat Rev Cancer 2006; 6: 425-436. https:/doi.org/10.1038/ $\underline{\operatorname{nrc1889}}$

[11] CUI Q, YANG S, YE P, TIAN E, SUN G et al. Downregulation of TLX induces TET3 expression and inhibits glioblastoma stem cell self-renewal and tumorigenesis. Nat Commun 2016; 7: 10637. https:/doi.org/10.1038/ncomms10637

[12] KE N, WANG X, XU X, ABASSI YA. The xCELLigence system for real-time and label-free monitoring of cell viability. Methods Mol Biol 2011; 740: 33-43. https:/doi. org/10.1007/978-1-61779-108-6 6

[13] KHO D, MACDONALD C, JOHNSON R, UNSWORTH CP, O`CARROLL SJ et al. Application of xCELLigence RTCA Biosensor Technology for Revealing the Profile and Window of Drug Responsiveness in Real Time. Biosensors (Basel) 2015; 5: 199-222. https:/doi.org/10.3390/bios5020199

[14] MARLINA S, SHU MH, ABUBAKAR S, ZANDI K. Development of a Real-Time Cell Analysing (RTCA) method as a fast and accurate screen for the selection of chikungunya virus 
replication inhibitors. Parasit Vectors 2015; 8: 579. https:/doi. org/10.1186/s13071-015-1104-y

[15] ZHANG CL, ZOU Y, YU RT, GAGE FH, EVANS RM. Nuclear receptor TLX prevents retinal dystrophy and recruits the corepressor atrophin1. Genes Dev 2006; 20: 1308-1320. https:/doi.org/10.1101/gad.1413606

[16] CHENG T, RODRIGUES N, SHEN H, YANG Y, DOMBKOWSKI D et al. Hematopoietic stem cell quiescence maintained by p21cip1/waf1. Science 2000; 287: 1804-1808. https:/doi.org/10.1126/science.287.5459.1804

[17] KIPPIN TE, MARTENS DJ, VAN-DER-KOOY D. p21 loss compromises the relative quiescence of forebrain stem cell proliferation leading to exhaustion of their proliferation capacity. Genes Dev 2005; 19: 756-767. https:/doi.org/10.1101/gad.1272305

[18] YILMAZ OH, VALDEZ R, THEISEN BK, GUO W, FERGUSON DO et al.Pten dependence distinguishes haematopoietic stem cells from leukaemia-initiating cells. Nature 2006; 441: 475-482. https:/doi.org/10.1038/nature04703

[19] TOThOVA Z, KOLLipaRA R, HUNTLY BJ, LEE BH, CASTRILLON DH et al. FoxOs are critical mediators of hematopoietic stem cell resistance to physiologic oxidative stress. Cell 2007; 128: 325-339. https:/doi.org/10.1016/j. cell.2007.01.003

[20] VAN-OS R, KAMMINGA LM, AUSEMA A, BYSTRYKH LV, DRAIJER DP et al. A Limited role for p21Cip1/Waf1 in maintaining normal hematopoietic stem cell functioning. Stem Cells 2007; 25: 836-843. https:/doi.org/10.1634/ stemcells.2006-0631

[21] SUN G, ALZAYADY K, STEWART R, YE P, YANG S et al.Histone demethylase LSD1 regulates neural stem cell proliferation. Mol Cell Biol 2010; 30: 1997-2005. https:/doi. org/10.1128/MCB.01116-09 